\begin{tabular}{|c|l|}
\hline Title & Beam-divergence deconvolution for diffractive imaging \\
\hline Author(s) & Kawahara, Kota; Gohara, Kazutoshi; Maehara, Yosuke; Dobashi, Takashi; Kamimura, Osamu \\
\hline Citation & $\begin{array}{l}\text { Physical Review B, 81(8),081404_1-081404.4 } \\
\text { https://doi.org/10.1103/PhysRevB.81.081404 }\end{array}$ \\
\hline Issue Date & 2010-02 \\
\hline Doc URL & http://hdl.handle.net/2115/45429 \\
\hline Rights & ○ 2010 The A merican Physical Society \\
\hline Type & article \\
\hline File Information & 20100209_Deconbolution_PRBe081404.pdf \\
\hline
\end{tabular}

Instructions for use 


\title{
Beam-divergence deconvolution for diffractive imaging
}

\author{
Kota Kawahara, ${ }^{1}$ Kazutoshi Gohara, ${ }^{1}$ Yosuke Maehara, ${ }^{1}$ Takashi Dobashi, ${ }^{2}$ and Osamu Kamimura ${ }^{2}$ \\ ${ }^{1}$ Division of Applied Physics, Graduate School of Engineering, Hokkaido University, Sapporo 063-8628, Japan \\ ${ }^{2}$ Central Research Laboratory, Hitachi Ltd., 1-280 Higashi-koigakubo Kokubunji-shi, Tokyo 185-8601, Japan
}

(Received 27 October 2009; revised manuscript received 27 December 2009; published 9 February 2010)

\begin{abstract}
This Rapid Communication presents a method of beam-divergence deconvolution for diffractive imaging. First, the detected diffraction intensity is formulated as a convolution between the diffraction intensity of parallel incident beams and the divergence of an incident beam. It is shown numerically that the convolution causes the reconstructed image to shrink and become blurred. Next, the algorithm of deconvolution used in the iterative Fourier phase retrieval method is applied to the convoluted diffraction intensity deteriorated by quantum noise. Numerical simulations show that the proposed algorithm recovers the deconvoluted diffraction intensity and improves the reconstructed image. Finally, the algorithm is applied to an electron-beam experiment to reconstruct a multiwall carbon nanotube. The results verified that the algorithm reduces the influence of beam divergence.
\end{abstract}

DOI: 10.1103/PhysRevB.81.081404

PACS number(s): 61.05.J-, 42.30.Rx

In recent years, diffractive imaging has developed into a powerful application for single-molecule imaging on nextgeneration synchrotrons. ${ }^{1-4}$ Using this method, one can reconstruct the structure of an object by achieving phase retrieval from a diffraction pattern in the Fourier domain. ${ }^{5,6}$ The application of these diffractive imaging techniques to x-ray scattering was advocated by Sayre. ${ }^{7}$ The first groundbreaking experiments, applying diffractive imaging to $\mathrm{x}$-ray diffraction, ${ }^{8}$ were made possible by recent technical developments: namely, incident beams with sufficient intensity and coherence, measurement systems having accurate linearity and wide dynamical range, and high-performance computation for calculating numeral iterations of the Fourier transform and inverse Fourier transform. Following this work, several novel experiments were performed using x-rays, including experiments on three-dimensional (3D) reconstruction, ${ }^{9,10}$ biospecimens, ${ }^{11-13}$ metallic materials, ${ }^{14}$ and experiments using a femtosecond soft-x-ray free-electron laser. ${ }^{2}$ However, in contrast to the large number of experiments with $\mathrm{x}$-rays, few applications have used electron beams. ${ }^{15-19}$ One exception is our previous experiment employing a prototype $20-\mathrm{kV}$-electron-beam microscope without a postspecimen lens to reconstruct a multiwall carbon nanotube (MWCNT) ${ }^{17}$ In this Rapid Communication, we discuss the influence of incident beam divergence on the diffraction pattern and demonstrate that the reconstructed size of an object is limited to the transverse-coherence length, which is affected by beam divergence. This influence has also been discussed in previous studies. ${ }^{15,20}$ In Ref. 20, numerical experiments showed the dependence of the reconstructed image in an $\mathrm{x}$-ray experiment on the transversecoherence length. In Ref. 15, experimental results using TEM showed numerically that the overall contrast of interference fringes is reduced due to the incoherent superposition of different Fourier intensities. On the other hand, the condition of spatial coherence based on the oversampling rate was defined. ${ }^{21}$ These works indicate that the necessary experimental condition of parallel electron beams is difficult to achieve using today's instruments. In this Rapid Communication, we present a fundamental solution for the influence of beam divergence. First, using a convolution model and nu- merical experiments, we confirm the influence of beam divergence on a reconstructed image. Next, an algorithm of deconvolution used in the iterative Fourier phase retrieval method is applied to the convoluted diffraction intensity deteriorated by quantum noise. Finally, the algorithm is applied to an electron-beam experiment of a MWCNT.

First, we formulated the convolution of the incident beam divergence. We assumed the diffraction of a light-element specimen (e.g., carbon) to be kinematical scattering. Therefore, the intensity $I_{0}(k)$ of the diffraction pattern that is radiated by an object $f(r)$, using parallel incident beams, is proportional to the square of the amplitude $|F(k)|$ of the Fourier transform $\mathcal{F}\{f(r)\}: I_{0}(k)=c|F(k)|^{2}=|\mathcal{F}\{f(r)\}|^{2}$. Henceforth, for simplicity, we will assume that the value of the proportional coefficient $c$ is 1 . The intensity $I_{\text {conv }}(k)$ convoluted by the incident beam divergence $h(k)$ of the point spread function is defined as follows: ${ }^{22}$

$$
I_{\text {conv }}(k)=\int_{-\infty}^{\infty} I_{0}\left(k-k^{\prime}\right) h\left(k^{\prime}\right) d k^{\prime} .
$$

Using Eq. (1) and a model object, we simulated the influence of convolution on a reconstructed image of diffractive imaging. Figure 1(a) shows the central area of the diffraction pattern $I_{0}$ obtained by the Fourier transform of the model object in Fig. 1( $\left.\mathrm{a}^{\prime}\right)$. The object, whose enlargement is shown in the inset of Fig. 1( $\left.a^{\prime}\right)$, is characterized by the four-wall structure of the MWCNT used in the experiment. ${ }^{17}$ In Fig. 1(a), we can clearly observe the fine structure of interference fringes corresponding to the vertical size of the object. We assume the incident beam divergence has a Gaussian distribution $h(k ; \sigma)=\frac{1}{\sqrt{2 \pi} \sigma} \exp \left(-\frac{k^{2}}{2 \sigma^{2}}\right)$. The parameter of the standard deviation $\sigma$ can be transformed to beam divergence $\alpha$ using the relation $\alpha=(p / L) \sigma$, where $L$ and $p$ are the camera length and the pixel size of a detector, respectively. Figures 1(b) and 1(c) show examples of convoluted diffraction patterns using $\sigma=1.5$ and $\sigma=6.0$, respectively. As the beam divergence increases, the patterns become broader. Figures $1 b^{\prime}$ and $1 c^{\prime}$ are corresponding reconstructed images. Comparing the three images in $\left(a^{\prime}\right),\left(b^{\prime}\right)$, and $\left(c^{\prime}\right)$, it can be seen that the object 


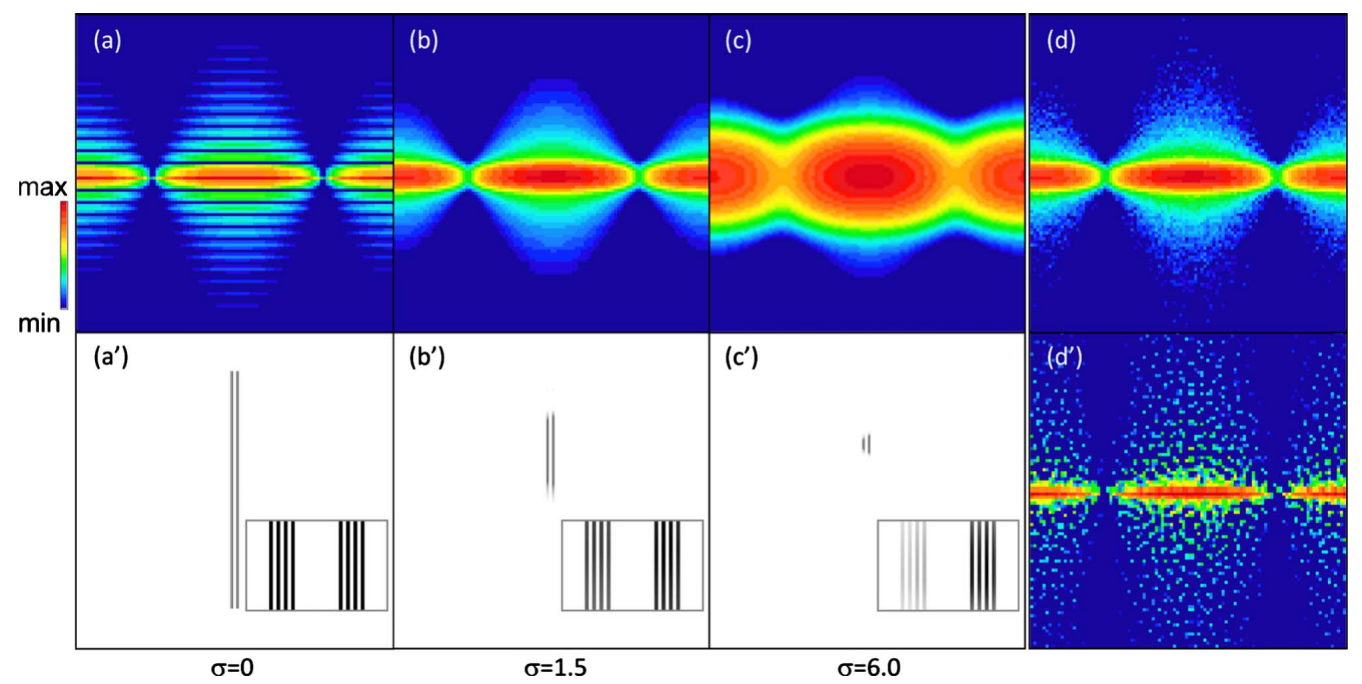

FIG. 1. (Color online) (a) The central area of the simulated diffraction pattern from the model object $\left(a^{\prime}\right)$. ( $\left.a^{\prime}\right)$ Structure of the object characterized by four walls. The inset is an enlargement of the central area of the object. The width between walls corresponds to 0.34 nm scaled by $0.095 \mathrm{~nm} /$ pixel. [(b) and (c)] The central area of a diffraction pattern convoluted by beam divergence: $\sigma=1.5,6.0$. $\left[\left(\mathrm{b}^{\prime}\right)\right.$ and $\left.\left(\mathrm{c}^{\prime}\right)\right]$ Images of the reconstructed object of diffractive imaging for $\sigma=1.5,6.0$. Diffractive imaging used the hybrid input-output (HIO) algorithm $(\beta=0.5,1000$ iterations) and the error-reduction (ER) algorithm (1000 iterations) tight support and real-positive constraints and started at a random phase. (d) The central area of the diffraction pattern including both noise and beam divergence: $\sigma=1.5$. ( $\left.\mathrm{d}^{\prime}\right)$ The diffraction pattern from direct deconvolution of (d) by the Richardson-Lucy algorithm (5000 iterations).

begins to shrink and become blurred not only in the vertical but also in the horizontal direction [see the inset in $\left.\left(\mathrm{c}^{\prime}\right)\right]$ as the beam divergence increases. The transverse-coherence lengths of $\sigma=1.5$ and 6.0 calculated by the relation $\lambda /(2 \alpha)$ using the experimental condition $p=25 \mu \mathrm{m}, L=0.57 \mathrm{~m}$, and $\lambda=0.00858 \mathrm{~nm}$ at $20 \mathrm{kV}$ are $65.2 \mathrm{~nm}$ and $16.3 \mathrm{~nm}$, respectively. The shrinking size indicates the same order of the transverse-coherence length. Consequently, these results demonstrate that the transverse coherence, which is affected by beam divergence, is proportional to the amount of shrinking in the size of the reconstructed image.

Second, we consider the conventional deconvolution of the intensity $I_{\text {noise }}$ contaminated by the quantum noise. When the beam-divergence distribution is known, to recover $I_{0}$ from Eq. (1), we can apply the deconvolution using the relations of the convolution theorem $\mathcal{F}\left\{I_{0} * h\right\}=\mathcal{F}\left\{I_{0}\right\} \mathcal{F}\{h\}$. Note that the asterisk denotes the convolution. By applying the inverse Fourier transform $\mathcal{F}^{-1}$, that is, the deconvolution, we can analytically obtain $I_{0}$ as follows:

$$
I_{0}=\mathcal{F}^{-1}\left\{\frac{\mathcal{F}\left\{I_{\text {conv }}\right\}}{\mathcal{F}\{h\}}\right\}, \quad(\mathcal{F}\{h\} \neq 0) .
$$

In an experiment without noise, applying the deconvolution of Eq. (2) is effective. However, no physical experiment can be conducted without errors such as the quantum noise, the thermal noise in the detector, and the scattering from gases in the vacuum of the electron optical system. In an experiment with noise, it is well known that obtaining an accurate $I_{0}$ by Eq. (2) is difficult due to the noise division by the neighborhood value of $\mathcal{F}\{h\}=0$. The problem of noise division on deconvolution has been richly studied using methods such as those of Wiener, Richardson-Lucy (RL), and maximum entropy. ${ }^{23}$
Next, using RL as an example and the same model object, we simulate the deconvolution's influence on the intensity of the diffraction pattern $I_{\text {noise }}$ contaminated by the quantum noise. Figure 1(d) shows a simulated diffraction pattern $I_{\text {conv }}$, $\sigma=1.5$ with the quantum noise. The intensity $n$ was calculated by Poisson distribution $P(n, \rho)=\frac{\rho^{n} e^{-\rho}}{n !}$, where $\rho$ is an expectation value, that is, the intensity without the noise at each point. The diffraction pattern including the Poisson noise is calculated as a random sample from the Poisson distribution with the expectation value of each point on the noise-free diffraction pattern. Figure $1(d)$ is obtained by the noise-free diffraction pattern of the total count $10^{7}$. The highintensity region (red and yellow area) is similar to the intensity without the noise, which is shown in Fig. 1(b). The low-intensity region (green and blue area) has noisy intensity, according to the influence of the Poisson noise. The algorithm of RL was formulated using $I_{\text {noise }}, h$, as follows:

$$
I_{\text {deconv }}(t+1)=I_{\text {deconv }}(t) \frac{I_{\text {noise }}}{I_{\text {deconv }}(t) * h} * \bar{h},
$$

where $t$ is the number of iterations and $\bar{h}$ is the conjugate function of $h$. Figure $1 \mathrm{~d}^{\prime}$ shows the deconvoluted intensity $I_{\text {deconv }}$ that was calculated by RL (5000 iterations) using $I_{\text {noise }}$ in Fig. $1(\mathrm{~d})$ and $h(k ; \sigma=1.5)$. No variation was observed in the diffraction pattern after 4000 , so we terminated the experiment after 5000 iterations. Although the high-intensity region (red and yellow area) is slightly recovered and has a fringelike shape, the low-intensity region (green and blue area) is not recovered due to the influence of the noise. As a result, we confirmed that it is difficult to achieve sufficient recovery for low intensity. Therefore, we need a useful deconvolution method for the diffractive imaging, using a dif- 


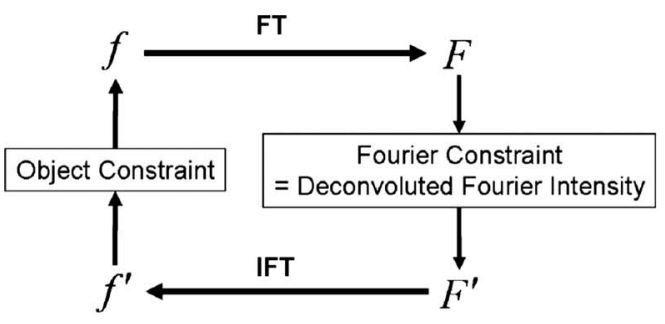

(a)

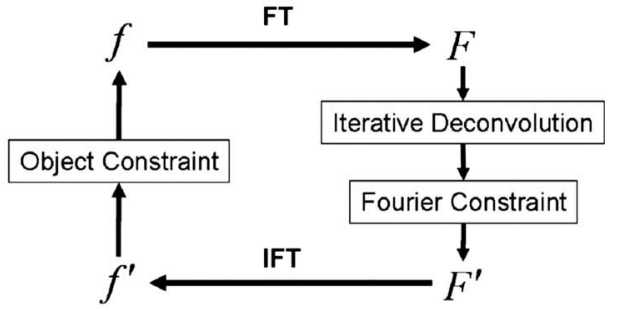

(b)

FIG. 2. Two usages of the deconvolution for diffractive imaging. (a) The algorithm is basically the conventional one, which uses a deconvoluted Fourier intensity as a Fourier constraint. (b) In the proposed algorithm, an iterative deconvolution is incorporated into the conventional iterative phase retrieval. The Fourier amplitude constraint uses the root of the deconvoluted intensity. The deconvolution utilizes an object-domain constraint.

fraction pattern deteriorated by Poisson noise.

Next, a deconvolution algorithm used in the iterative Fourier phase retrieval method is applied to the convoluted diffraction intensity deteriorated by quantum noise. In addition, we demonstrate the effectiveness of the algorithm for improving a reconstructed image. Figures 2(a) and 2(b) show the algorithm of the conventional deconvolution used for Fig. $1 \mathrm{~d}^{\prime}$ and the algorithm of the proposed deconvolution, respectively. The conventional algorithm (a) is the conventional iterative phasing algorithm, ${ }^{5,6}$ using a deconvoluted Fourier intensity as a Fourier constraint. In the proposed algorithm (b), an iterative deconvolution algorithm, such as Richardson-Lucy and maximum entropy, is used in the conventional iterative phasing algorithm. If the incident beam divergence is approximated as the delta function in this proposed algorithm form, the form corresponds to the conventional phasing method.

Using the proposed algorithm together with the RL algorithm of Eq. (3) and the same model object in Fig. 1, we simulate the deconvolution's effect on improving a reconstructed image in the case of a large amount of noise. Figure 3 (a) is a graph of $R$-factor dependency on the number of iterations using the proposed algorithm. The $R$ factor is defined by $R=\frac{\Sigma_{k}|F(k)|-\left|F_{o b s}(k)\right| \mid}{\Sigma_{k}\left|F_{o b s}(k)\right|}$. In the graph, three diffraction patterns were inserted at intermediate stages 2000, 5500, and 7000. The graph and patterns show how the original diffraction pattern that had deteriorated by beam divergence gradually improves its quality until it is almost the same as that without the deterioration. Figure 3(b) shows the deconvo- luted intensity at the final 10000 iterations. The results demonstrate that the diffraction pattern in Fig. 3(b) is similar to the ideal pattern shown in Fig. 1(a). We confirmed the effect of the proposed deconvolution by comparing the line profiles of the diffraction pattern in Figs. 1(a), 1(d), and 3(b) [the comparison is shown in Fig. 3(c)]. Figure 3(d) shows a reconstructed image of the proposed algorithm. The reconstructed image indicates that the convolution's influence, i.e., the "shrinking," is less than that in Fig. 1 b'. In addition, the wall structures of the MWCNT were correctly reconstructed, as shown in the inset of Fig. 3(d). These results clearly show that the proposed algorithm for diffractive imaging was effective.

Finally, we demonstrate the effect of the proposed algorithm on a practical problem. We applied the proposed algorithm to diffractive imaging using the diffraction pattern measured from a MWCNT. ${ }^{17}$ The experimental setup was as follows: the acceleration voltage was $20 \mathrm{kV}$, the camera length was $0.57 \mathrm{~m}$, and the detector was an imaging plate with a trimming size of $2048 \times 2048$ pixels. After the incident beam intensity was measured without a specimen, the intensity was subtracted from the obtained diffraction pattern. Figure 4(a) shows the distribution of the measured incident beams. We assumed a uniform contour plot $\sigma=\sigma_{x}$ $=\sigma_{y}$. The $\sigma$ on the beam distribution was estimated at $1.55 \pm 0.15$ pixels $(0.068 \pm 0.007 \mathrm{mrad})$. Figure $4(\mathrm{~b})$ shows the central area of the raw experimental diffraction pattern with the same scale as in Fig. 1(a). A nonsymmetrical broad pattern into the upper and lower directions is observed. The nonsymmetry might be due to scattering noises from the

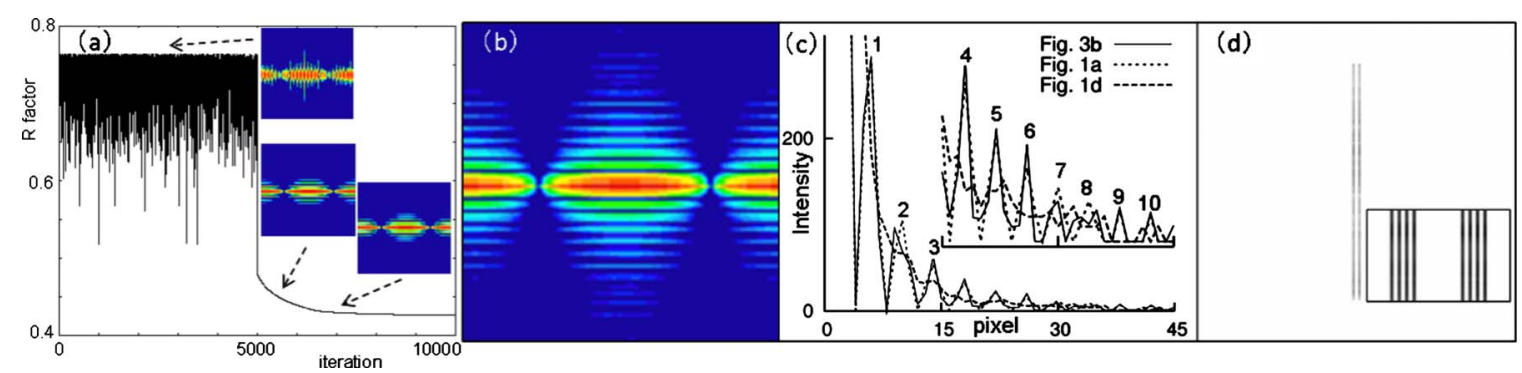

FIG. 3. (Color online) (a) The graph of $R$-factor dependency on the number of iterations using the proposed algorithm. Three diffraction patterns were inserted at intermediate stages 2000, 5500, and 7000. (b) The diffraction pattern from the application of the proposed algorithm to $I_{\text {noise }}, \sigma=1.5$ in Fig. 1(d). (c) Comparison of profiles along central vertical lines in Figs. 1(a), 1(d), and 3(b). (d) An image reconstructed from (b). Diffractive imaging using HIO with RL (real positive, $\beta=0.5$, 5000 iterations) and ER with RL (5000 iterations) tight support and real-positive constraints and started at a random phase. 


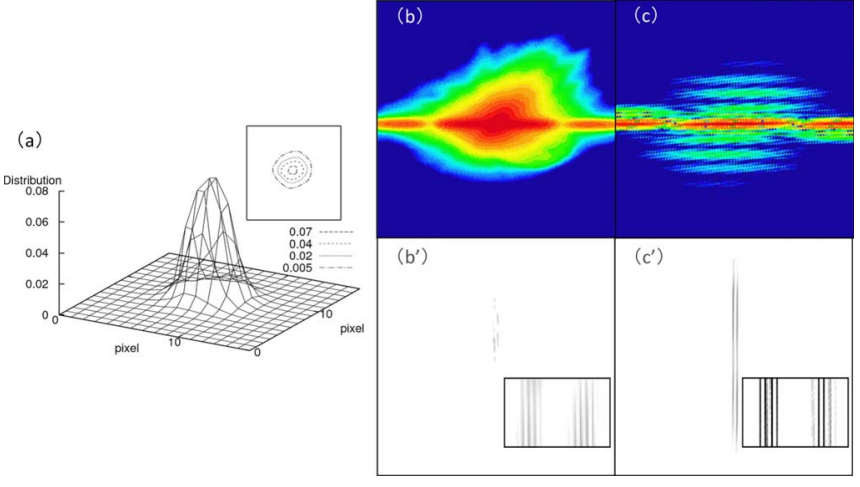

FIG. 4. (Color online) (a) Distribution of incident beam divergence (3D and contour plot). (b) The raw experimental diffraction pattern with the same scale as in Fig. 1(a). ( $\left.\mathrm{b}^{\prime}\right)$ A reconstructed image using the raw diffraction pattern. (c) The diffraction pattern after the proposed deconvolution was applied. ( $\left.\mathrm{c}^{\prime}\right)$ A reconstructed image using the deconvoluted diffraction pattern. Diffractive imaging using HIO with RL ( $\beta=0.5,5000$ iterations) and ER with RL (5000 iterations); support $2048 \times 50$ pixels real-positive constraints and started at a random phase. Central missing data were calculated using a diffraction pattern.

edges of meshes that supported the target MWCNT. Figure $4 b^{\prime}$ is an image reconstructed using Fig. 4(b) before the deconvolution was applied. The influence of shrinking the reconstructed area in the vertical direction corresponded to the transverse-coherence length $63.1 \mathrm{~nm}$ calculated by the relation $\lambda /(2 \alpha)$. The reconstructed wall structure in the horizontal direction [see the inset in Fig. 4b'] was observed. Figure 4(c) shows the central area of the diffraction pattern after the proposed deconvolution was applied. The fine structures of the interference fringes are clearly observed. The intervals between fringes are slightly wider than those in Figs. 1(a) and 3(b). Figure $4 c^{\prime}$ is a reconstructed image using Fig. 4(c). Although the blurred region remained, the reconstructed area in the vertical direction was expanded. In addition, the wall structures in the horizontal direction [see the inset in Fig. $\left.4 c^{\prime}\right]$ were improved. Thus, we demonstrated the efficacy of the proposed deconvolution method by both numerical experiments and practical measurements.

In this Rapid Communication, we examined the convolution and deconvolution of beam divergence for diffractive imaging. We confirmed that the influence of beam divergence on a reconstructed image resulted in the shrinkage of the area of the reconstructed structure. For the problems of beam divergence and quantum noise, we proposed a deconvolution algorithm for diffractive imaging. The algorithm reduced the noise's effect and improved the reconstructed image. The algorithm was demonstrated by applying it to the diffraction pattern obtained from a MWCNT. In our calculations, we used tight support, in which the support is the same size as the target object. In this condition, the convergences occurred because an oversampling ratio between target area and total detected area is about 40. Actually, similar images using different initial images were obtained at the final stage, although the intermediate images generally depend on the initial conditions. In this study, we assumed that the beamdivergence distribution was a priori knowledge; however, in the case of imperfect distribution, it is necessary to apply a blind deconvolution method to diffractive imaging. ${ }^{24,25} \mathrm{We}$ also verified the maximum entropy algorithm instead of using the Richardson-Lucy algorithm. Although we did not recognize any crucial difference between these methods, further research will be needed to examine these algorithms and their applications to various experimental data.
${ }^{1}$ R. Neutze et al., Nature (London) 406, 752 (2000).

${ }^{2}$ H. N. Chapman et al., Nat. Phys. 2, 839 (2006).

${ }^{3}$ M. Bogan et al., Nano Lett. 8, 310 (2008).

${ }^{4}$ M. M. Murnane and J. Miao, Nature (London) 460, 1088 (2009).

${ }^{5}$ R. W. Gerchberg and W. O. Saxton, Optik (Stuttgart) 34, 275 (1971).

${ }^{6}$ J. R. Fienup, Appl. Opt. 21, 2758 (1982).

${ }^{7}$ D. Sayre, Imaging Processes and Coherence in Physics, Lecture Notes in Physics, edited by M. Schlenker et al. (Springer-Verlag, Berlin, 1980) 112, 229.

${ }^{8}$ J. Miao et al., Nature (London) 400, 342 (1999).

${ }^{9}$ J. Miao et al., Phys. Rev. Lett. 89, 088303 (2002).

${ }^{10}$ H. Chapman et al., J. Opt. Soc. Am. A Opt. Image Sci. Vis 23, 1179 (2006).

${ }^{11}$ J. Miao et al., Proc. Natl. Acad. Sci. U.S.A. 100, 110 (2003).

${ }^{12}$ P. Thibault et al., Acta Crystallogr., Sect. A: Found. Crystallogr.
62, 248 (2006).

${ }^{13}$ H. Jiang et al., Phys. Rev. Lett. 100, 038103 (2008).

${ }^{14}$ Y. Takahashi et al., Appl. Phys. Lett. 90, 184105 (2007).

${ }^{15}$ U. Weierstall et al., Ultramicroscopy 90, 171 (2002).

${ }^{16}$ J. M. Zuo et al., Science 300, 1419 (2003).

${ }^{17}$ O. Kamimura et al., Appl. Phys. Lett. 92, 024106 (2008).

${ }^{18}$ S. Morishita et al., Appl. Phys. Lett. 93, 183103 (2008).

${ }^{19}$ W. Huang et al., Nat. Phys. 5, 129 (2009).

${ }^{20}$ I. A. Vartanyants and I. K. Robinson, J. Phys.: Condens. Matter 13, 10593 (2001).

${ }^{21}$ J. Miao et al., Phys. Rev. B 67, 174104 (2003).

${ }^{22}$ W. E. McBride et al., Ultramicroscopy 76, 115 (1999).

${ }^{23}$ P. Jansson, Deconvolution of Images and Spectra (Academic Press, New York, 1997).

${ }^{24}$ J. Seldin and J. Fienup, J. Opt. Soc. Am. A 7, 428 (1990).

${ }^{25}$ D. Fish et al., J. Opt. Soc. Am. A Opt. Image Sci. Vis 12, 58 (1995). 